Artículo científico

Volumen 31(3):679-694. Septiembre-diciembre, 2020 e-ISSN 2215-3608, doi:10.15517/am.v31i3.39059 http://www.revistas.ucr.ac.cr/index.php/agromeso

\title{
Caracterización de la población bovina cebú con certificado de registro genealógico en Costa Rica ${ }^{1}$
}

\section{Characterization of the Zebu cattle population with a certificate of genealogical registration in Costa Rica}

\author{
Marilyn Vásquez-Loaiza², Roger Molina-Coto
}

1 Recepción: 18 de setiembre, 2019. Aceptación: 30 de marzo, 2020. Este trabajo formó parte del proyecto de graduación de la primera autora para la licenciatura en Ingeniería Agronómica con énfasis en Zootecnia de la Universidad de Costa Rica.

2 Asociación de Criadores de Ganado Cebú de Costa Rica. San José, Costa Rica. marilyntatiana.vasquez@gmail.com (https://orcid.org/00000002-7267-603X).

3 Universidad de Costa Rica, Facultad de Ciencias Agroalimentarias, Escuela de Zootecnia, San José, Costa Rica. roger.molina@ucr.ac.cr (https://orcid.org/0000-0003-3844-2587).

\section{Resumen}

Introducción. Existe escasa información sobre ganado puro con certificado genealógico en Costa Rica. Es necesario conocer el estado de esta población para dirigir esfuerzos en la mejora productiva del ganado cebú. Objetivo. Actualizar la base de datos de la Asociación de Criadores de Ganado Cebú de Costa Rica (ASOCEBÚ), caracterizar dicha población y analizar el uso de los machos de la raza Brahman gris en el país. Materiales y métodos. Se realizó un inventario en 121 ganaderías para actualizar la base de datos de la Asociación al año 2018. La información se clasificó por raza, sexo, edad, total de animales, criadores, región socio-económica, provincia y cantón. Se determinó la frecuencia de uso de reproductores Brahman gris. Resultados. Se contabilizaron 10995 animales puros, distribuidos en seis razas (Brahman gris, Brahman rojo, Nelore, Gyr, Guzerat e Indubrasil). La raza Brahman gris predominó con un 74,5 \% de la población y 76,8 \% del total de criadores. La región Chorotega presentó la mayor población inscrita $(49,5 \%)$ y el mayor número de criadores $(37,2 \%)$. El 55,8 \% de la población total provino de diez criadores, sin embargo, el mayor número de ganaderías correspondió a fincas con pocos animales. En la raza Brahman gris, el 60,8 \% de la población provino de toros nacionales y el 39,2 \% del extranjero. Conclusión. Los cebuinos puros registrados se distribuyen en todo el país. Se debe poner atención al manejo genético para minimizar la consanguidad. Se recomienda a los encargados de censos y encuestas, incluir la población de razas puras dentro de las evaluaciones.

Palabras clave: Bos indicus, pedigrí, sementales, raza pura.

\begin{abstract}
Introduction. There is not much information on pure cattle with a genealogical certificate in Costa Rica. It is necessary to know the status of this population to direct efforts towards the productive improvement of the Zebu cattle breeds. Objective. To update the database of the Costa Rican Cebu Cattle Breeders Association (ASOCEBÚ), characterize this population and analyze the use of males of the gray Brahman breed in the country. Materials and
\end{abstract}


methods. An inventory was done in 121 herds to update the Association's database to 2018. The information was sorted by breed, sex, age, total animals, breeders, socio-economic region, province, and canton. The frequency of use of gray Brahman breeders was determined. Results. A total of 10995 pure animals were counted, distributed in six breeds (gray Brahman, red Brahman, Nelore, Gyr, Guzerat, and Indubrasil). The gray Brahman breed predominated with $74.5 \%$ of the population and $76.8 \%$ of the total of breeders. The Chorotega region had the highest registered population (49.5 $\%)$ and the highest number of breeders (37.2\%). $55.8 \%$ of the total population came from ten breeders, however, the largest number of herds corresponds to farms with few animals. In the gray Brahman breed, $60.8 \%$ of the population comes from domestic bulls, and 39.2\% from other countries. Conclusion. Registered pure zebu cattle are distributed throughout the whole country. Attention should be put to genetic management to minimize inbreeding. Census and survey managers are encouraged to include the purebred populations in their assessments.

Keywords: Bos indicus, pedigree, sires, pure breed.

\section{Introducción}

La ganadería bovina en el mundo cuenta con lo que se ha denominado, dos subespecies, Bos taurus y Bos indicus, entre estas subespecies existen cientos de razas (Loftus et al., 1994). El ganado cebú (Bos indicus) predomina en zonas de clima tropical y subtropical (Takeshima et al., 2018), por tanto, están presentes en Costa Rica.

Los datos sobre población bovina en Costa Rica, han sido recopilados a través de los años. En el año 1973 el Censo Agropecuario reportó 1693000 cabezas de animales, las encuestas ganaderas de 1982 y 1988 indicaron contar con 2276000 y 2190000 animales, respectivamente (CORFOGA, 2000); recientemente se cuenta con los datos del Censo Ganadero realizado en el año 2000 de manera conjunta entre la Corporación de Fomento Ganadero (CORFOGA), el Ministerio de Agricultura y Ganadería y el Programa de Erradicación del Gusano Barrenador, el cual contabilizó 1358209 cabezas de animales. Además, CORFOGA realizó la Encuesta Ganadera en el año 2012 (1 575779 animales) y el Instituto Nacional de Estadística y Censo elaboró el VI Censo Nacional Agropecuario en el año 2014 (1 278817 animales).

La información generada en estos censos o encuestas ha caracterizado los sistemas de producción de carne, leche y doble propósito en la ganadería de cría comercial, haciendo referencia a animales con algún porcentaje de sangre cebuína, y en el caso de la encuesta ganadera del año 2012, generó información sobre el componente racial, se incluyó de manera específica la raza Brahman, se demostró que se encontraba presente en el 39,7 \% del total de fincas dedicadas a la actividad ganadera (fincas de leche, carne y doble propósito). Sin embargo, en estos estudios no se indica si los datos corresponden a animales puros y mucho menos si estos contaban con certificado de registro genealógico.

El registro genealógico de ganado en Costa Rica inició en el año de 1946 bajo la responsabilidad de la Secretaría de Agricultura (Quirós, 2006); sin embargo, desde la fecha y hasta el año de 1981 todos los animales se registraban como cebú sin distinción de raza (ASOCEBÚ, 2020). En el año 1989, el Ministerio de Agricultura y Ganadería (MAG), traspasó el Registro Genealógico de Ganado a las Asociaciones de Productores y Criadores de Ganado de la República (decreto 19400-MAG) (Presidente de la República y MAG, 1989), el 16 de setiembre de 1994, por medio del decreto 23680-MAG inició funciones la Asociación de Criadores de Ganado Cebú de Costa Rica (ASOCEBÚ).

Desde el traslado del registro por parte del Ministerio de Agricultura y Ganadería hacia ASOCEBÚ, el control de la genealogía de todas las razas cebuinas y sus cruces reportó desde esta fecha y hasta el 2017 un total de 68 
052 animales inscritos de la raza Brahman Gris, 27148 Indubrasil, 13623 Nelore, 9746 Gyr y 3889 Brahman Rojo (ASOCEBÚ, 2017). Estos datos representaron el total de animales inscritos, sin reflejar la población real que conformaba el hato puro nacional.

La raza Brahman es la de mayor crianza en Costa Rica, se estima que abarca cerca del $80 \%$ del total de población registrada en la Asociación de Criadores de Ganado Cebú de Costa Rica (ASOCEBÚ, 2017). Según datos de Madrigal y Fallas (2013), la raza Brahman también era la raza predominante en los sistemas de producción de carne a nivel comercial, y abarcó el 77,7 \% del total de razas presentes. Una de las razones por las cuales existe esta población importante de sangre cebuína en la ganadería de carne nacional, se atribuye a la mayor capacidad de adaptación al trópico en comparación con animales Bos taurus (Vélez, 2016).

Es escasa la información sobre las razas Bos indicus con certificado de registro genealógico utilizadas en Costa Rica. Previo a la realización del inventario, ASOCEBÚ no contaba con datos recientes sobre la cantidad de animales inscritos que conforman el hato de ganado puro nacional, por tanto, había desconocimiento sobre la caracterización de la población actual de las razas cebuinas en Costa Rica. Investigaciones previas alertaron sobre consanguinidad en el hato Brahman puro (Campos et al., 2018) y en otros grupos raciales puros en el país (Martínez et al., 2015). El registro genealógico es una herramienta fundamental para promover el mejoramiento genético, al contener información genealógica y de población de cada una de las razas. Países líderes en el desarrollo de las razas Bos indicus como Brasil, Colombia y Estados Unidos (en el caso de la raza Brahman para este último), generan parámetros de los animales puros e inscritos que ayudan para la toma de decisiones de los ganaderos y organizaciones.

Esta investigación tuvo como objetivo actualizar la base de datos de la Asociación de Criadores de Ganado Cebú de Costa Rica (ASOCEBÚ), caracterizar dicha población y analizar el uso de los machos de la raza Brahman gris en el país.

\section{Materiales y métodos}

Para la determinación de la población Bos indicus se utilizaron los datos generados en el inventario físico nacional realizado en Costa Rica por la Asociación de Criadores de Ganado Cebú de Costa Rica (ASOCEBÚ), el cual consistió en un censo realizado en 121 fincas de un total de 126 ganaderías activas a la fecha de la realización del estudio, con más de un $96 \%$ de participación de las ganaderías.

La primera etapa consistió en definir la población teórica, se utilizó como referencias las inscripciones de nacimientos realizadas en ASOCEBÚ durante el periodo de enero del 2010 a diciembre del año 2017; para efectos del estudio se definió como ganadería activa aquellas que inscribieron al menos un animal durante este periodo.

La segunda fase fue la coordinación de las visitas a las fincas por zona geográfica para la recolección de datos. Antes de cada inspección se generó un informe de animales inscritos por criador, el cual contenía información de la identificación del animal, raza, edad al día de la visita, sexo e identificación de la madre. Esta información permitió verificar que los animales presentados por el criador durante la visita coincidieran con los datos de los animales inscritos en ASOCEBÚ. Durante estas visitas se validó información sobre la cantidad de animales puros, inscritos y activos, así como la cantidad de animales por raza (Brahman gris, Brahman rojo, Nelore, Gyr, Guzerat e Indubrasil) presentes en el país. Además, se actualizó la información de ubicación geográfica referente a la provincia, cantón y distrito.

La tercera fase fue el análisis de la información recopilada en campo, la edición de datos y la actualización del Registro Nacional Ganadero de ASOCEBÚ, mediante la digitación de los datos obtenidos en el censo. Esta actualización consistió en inactivar en el software cada uno de los animales ausentes al momento de la visita, los mismos fueron definidos como fuera de inventario 2017-2018, por lo que se excluyeron del inventario de cada uno de los criadores en el Registro Nacional Ganadero. 
En el caso específico de los animales que fueron vendidos para la cría de animales puros, se realizó el seguimiento a través de los traspasos o cambios de propietario registrados en ASOCEBÚ y fueron inspeccionados en las fincas de los nuevos propietarios. Los reproductores que fueron vendidos a fincas de cría comercial no formaron parte del estudio.

Una vez finalizada la actualización de la base de datos, se generó un informe final de la población activa a través del Registro Nacional Ganadero. El análisis de la información se llevó a cabo con base en estadística descriptiva en hojas de Microsoft Office Excel® para las variables de raza, sexo y edad. Adicionalmente, se generó información sobre el total de animales y criadores por región, provincia y cantón; número de criadores por raza y distribución de la población activa de acuerdo con los rangos de edad. Debido al mayor porcentaje de la población Brahman dentro de la población total de ganado cebuino, se determinó la frecuencia de uso de reproductores dentro de la población activa en dicha raza. Se hizo uso de la base de datos actualizada del Registro Nacional Ganadero de ASOCEBÚ. En total, se analizaron 8191 registros de nacimientos de la raza Brahman inscritos en el periodo de enero del año 2003 a marzo del 2018, pertenecientes a 93 criadores distribuidos en las diferentes regiones del país. Adicionalmente, se analizó la genealogía de los primeros cinco animales más utilizados a nivel nacional con el fin de determinar sus líneas de sangre.

\section{Resultados}

\section{Población de Bos indicus y distribución racial en el país}

Hasta el mes de marzo del año 2018, se contabilizó una población activa de 10995 animales Bos indicus con certificado de registro genealógico, en dicha fecha se realizó la finalización de actualización de la base de datos. La distribución porcentual y absoluta de la población de animales de acuerdo con cada una de las razas obtenidas en el estudio se muestra en la Figura 1.
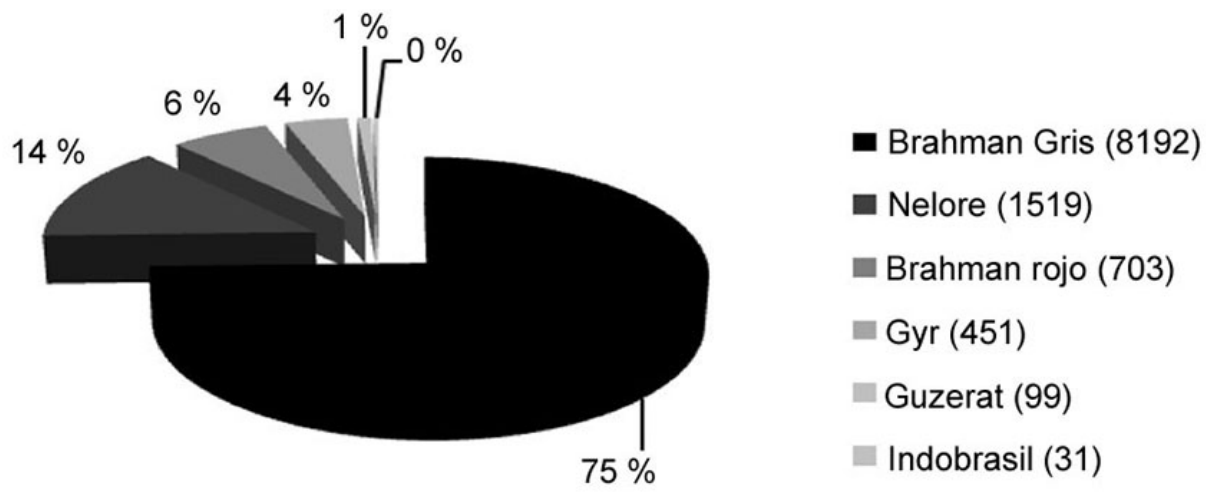

Figura 1. Distribución porcentual de la población activa de Bos indicus con certificado de registro geneológico en Costa Rica (valores absolutos entre paréntesis). San José, Costa Rica. 2018.

Figure 1. Percentage distribution of the active population of Bos indicus with a certificate of genealogical registration in Costa Rica (absolute values in parentheses). San Jose, Costa Rica. 2018. 
La raza Brahman gris fue la raza predominante, dentro de la población total de animales con registro genealógico representó el 74,5\%, seguida por la raza Nelore con el 13,8 \%, el tercer grupo con mayor número de animales correspondió a la raza Brahman rojo con un 6,4\%. Por su parte, la raza de menor población activa fue la Indobrasil con 31 animales que correspondió al $0,3 \%$ de la población total.

La distribución porcentual y absoluta de la población activa registrada en ASOCEBÚ, de acuerdo con las variables de raza (Brahman gris, Nelore, Brahman rojo, Gyr, Guzerat e Indobrasil) y sexo, así como el número de criadores por raza, se muestran en el Cuadro 1.

Cuadro 1. Población activa de animales Bos indicus con certificado de registro genealógico, según raza, sexo y número de criadores al mes de marzo de 2018. San José, Costa Rica. 2018.

Table 1. Active population of Bos indicus animals with genealogical certificate, according to breed, sex, and number of breeders as of March 2018. San Jose, Costa Rica. 2018.

\begin{tabular}{lcccc}
\hline Raza & Sexo & Número de animales & Distribución porcentual & Número de criadores \\
\hline Brahman gris & Hembras & 5676 & 69,3 & 93 \\
Nelore & Machos & 2516 & 30,7 & \\
& Hembras & 1171 & 77,1 & 21 \\
Brahman rojo & Machos & 348 & 22,9 & 28 \\
& Hembras & 519 & 73,8 & \\
Gyr & Machos & 184 & 26,2 & 21 \\
& Hembras & 340 & 75,4 & \\
Guzerat & Machos & 111 & 24,6 & 4 \\
& Hembras & 79 & 79,8 & \\
Indobrasil & Machos & 20 & 20,2 & 3 \\
& Hembras & 26 & 83,9 & \\
\hline
\end{tabular}

\section{Población de Bos indicus y su distribución en el país por número de criadores}

La cantidad de criadores que registraron la raza Brahman gris representó un 76,8 \% de las fincas que fueron inventariadas, estos criadores se dedicaban a la cría de la raza Brahman gris de manera exclusiva o conjunta con otras razas. Asimismo, a pesar de que la raza Gyr, en cuanto a número de animales, alcanzó apenas el $4 \%$ de la población, en términos de la cantidad de criadores aportó un porcentaje de 17,3\%.

La población animal se distribuyó en rangos por número de animales con el fin de determinar la distribución de la población animal en los criadores (Cuadro 2).

Según los datos, el 55,8 \% del total de animales de la población activa provino de diez criadores y se ubicaron dentro del rango de ganaderías con más de 300 cabezas de animales; seguido por el 19,2 \% de la población, que perteneció a dieciocho criadores y se encontró en el rango de 85 a 174 cabezas de animales. El menor porcentaje de la población (2,6\%) correspondió al mayor número de criadores, ubicados en el rango de 1 a 15 cabezas de animales. 
Cuadro 2. Distribución de criadores y población Bos indicus de acuerdo al número de animales. San José, Costa Rica. 2018.

Table 2. Distribution of breeders and Bos indicus population according to the number of animals. San Jose, Costa Rica. 2018.

\begin{tabular}{lcccc}
\hline \multirow{2}{*}{$\begin{array}{l}\text { Rango del número de animales } \\
\text { por criador }\end{array}$} & \multicolumn{2}{c}{ Total criadores } & \multicolumn{2}{c}{ Total animales } \\
\cline { 2 - 5 } & Cantidad & \% & Cantidad & \% \\
\hline$<15$ & 43 & 35,5 & 285 & 2,6 \\
15 a 30 & 19 & 15,7 & 385 & 3,5 \\
31 a 84 & 27 & 22,3 & 1233 & 11,2 \\
85 a 174 & 18 & 14,9 & 2116 & 19,2 \\
175 a 299 & 4 & 3,3 & 846 & 7,7 \\
$\geq 300$ & 10 & 8,3 & 6130 & 55,8 \\
\hline Total general & $\mathbf{1 2 1}$ & $\mathbf{1 0 0 , 0}$ & $\mathbf{1 0 ~ 9 9 5}$ & $\mathbf{1 0 0 , 0}$ \\
\hline
\end{tabular}

\section{Población de Bos indicus y su distribución en el país por división política}

La cantidad de animales Bos indicus por raza de acuerdo con cada una de las provincias y sus respectivos cantones se muestran en el Cuadro 3.

Los cantones de Cañas, Pococí, Abangares, Perez Zeledón, Liberia y San Carlos fueron los de mayor número de población, y como provincia, Guanacaste fue la de mayor población para todas las razas, excepto por la raza Gyr y Brahman rojo predominaron en Heredia y Alajuela, respectivamente.

\section{Población de Bos indicus y su distribución en el país por región socio-económica}

Costa Rica se divide en seis regiones socio-económicas, en el Cuadro 4, se muestra la distribución de animales Bos indicus según raza para cada región socio-económica del país. Al analizar los datos por región, la Chorotega fue la región con mayor cantidad de criadores (45 en total), en donde el promedio de animales por criador fue el más alto a nivel nacional (121), seguida por la región Huetar Atlántica con 23 criadores y un promedio de 89 animales por criador. Las regiones Brunca y Huetar Norte contaron con 15 criadores por región para un promedio de 89 y 76 animales por criador, respectivamente. La región con menor promedio de animales por criador (13) fue la Central (36 animales), por su parte la región con el menor número de criadores fue la Pacífico Central (10), con un promedio de 54 animales por criador.

\section{Uso de los machos reproductores de la raza Brahman gris con certificado genealógico en Costa Rica}

La población activa (8192 animales) de la raza Brahman gris con certificado de registro genealógico provino de 738 machos progenitores, la mayoría de estos toros contaban con registro nacional de raza (Cuadro 5). De los toros reproductores inscritos en el extranjero, el origen principal fue Estados Unidos y en menor proporción países como Brasil, Panamá, Guatemala y Canadá.

El análisis de la frecuencia de uso de los reproductores dentro de la población activa de la raza Brahman gris demostró que el 35,8 \% de la población total proviene de 38 toros, los cuales de manera individual presentaron, al menos, una descendencia superior al 0,5\% de la población, siendo en total una progenie de 2936 animales (Cuadro 6). Respecto al origen de estos 38 toros, 19 contaban con registro nacional y 19 correspondían a registro extranjero. 
Cuadro 3. Distribución de animales activos Bos indicus con certificado de registro genealógico en Costa Rica al mes de marzo 2018, según raza, cantón y provincia. San José, Costa Rica. 2018.

Table 3. Distribution of Bos indicus active animals with a genealogical record certificate in Costa Rica as of March 2018, according to breed, canton, and province. San Jose, Costa Rica. 2018.

\begin{tabular}{|c|c|c|c|c|c|c|c|}
\hline $\begin{array}{l}\text { Cantón/ } \\
\text { Provincia }\end{array}$ & Brahman gris & Nelore & $\begin{array}{l}\text { Brahman } \\
\text { rojo }\end{array}$ & Gyr & Guzerat & Indobrasil & Total \\
\hline Alajuela & 195 & 1 & 118 & 0 & 0 & 0 & 314 \\
\hline Grecia & 16 & 0 & 0 & 0 & 0 & 0 & 16 \\
\hline Los Chiles & 5 & 0 & 3 & 0 & 0 & 0 & 8 \\
\hline Orotina & 25 & 0 & 2 & 0 & 0 & 0 & 27 \\
\hline San Carlos & 518 & 34 & 76 & 98 & 0 & 14 & 740 \\
\hline Upala & 232 & 0 & 160 & 0 & 0 & 0 & 392 \\
\hline San Ramón & 32 & 0 & 0 & 0 & 0 & 0 & 32 \\
\hline Alajuela & 1023 & 35 & 359 & 98 & $\mathbf{0}$ & 14 & 1529 \\
\hline Cartago & 3 & 0 & 0 & 2 & 0 & 0 & 0 \\
\hline Paraíso & 0 & 3 & 0 & 0 & 0 & 0 & 0 \\
\hline Turrialba & 13 & 0 & 0 & 0 & 0 & 0 & 0 \\
\hline Cartago & 16 & 3 & $\mathbf{0}$ & 2 & $\mathbf{0}$ & $\mathbf{0}$ & 21 \\
\hline Abangares & 449 & 718 & 14 & 0 & 57 & 7 & 1245 \\
\hline Bagaces & 51 & 0 & 0 & 0 & 38 & 0 & 89 \\
\hline Cañas & 2221 & 398 & 205 & 43 & 0 & 0 & 2867 \\
\hline Carrillo & 30 & 9 & 0 & 0 & 0 & 0 & 39 \\
\hline La Cruz & 42 & 0 & 0 & 0 & 0 & 0 & 42 \\
\hline Liberia & 899 & 0 & 30 & 34 & 3 & 0 & 966 \\
\hline Santa Cruz & 191 & 0 & 0 & 10 & 0 & 0 & 201 \\
\hline Guanacaste & 3883 & 1125 & 249 & 87 & 98 & 7 & 5449 \\
\hline Sarapiquí & 140 & 1 & 40 & 144 & 0 & 0 & 325 \\
\hline Heredia & 140 & 1 & 40 & 144 & 0 & 0 & 325 \\
\hline Guácimo & 11 & 0 & 0 & 0 & 0 & 0 & 0 \\
\hline Pococí & 1430 & 200 & 16 & 60 & 1 & 0 & 1707 \\
\hline Limón & 1441 & 200 & 16 & 60 & 1 & 0 & 1718 \\
\hline Coto Brus & 160 & 0 & 0 & 0 & 0 & 0 & 160 \\
\hline Esparza & 85 & 0 & 0 & 47 & 0 & 0 & 132 \\
\hline Golfito & 20 & 20 & 0 & 0 & 0 & 0 & 40 \\
\hline Osa & 32 & 0 & 0 & 0 & 0 & 0 & 32 \\
\hline Puntarenas & 133 & 0 & 6 & 7 & 0 & 0 & 146 \\
\hline Parrita & 233 & 0 & 6 & 0 & 0 & 0 & 239 \\
\hline Puntarenas & 663 & 20 & 12 & 54 & 0 & 0 & 749 \\
\hline Acosta & 0 & 10 & 0 & 0 & 0 & 0 & 10 \\
\hline Goicoechea & 3 & 0 & 0 & 0 & 0 & 0 & 3 \\
\hline Mora & 0 & 0 & 0 & 6 & 0 & 10 & 16 \\
\hline Pérez Zeledón & 1017 & 2 & 96 & 0 & 0 & 0 & 1115 \\
\hline Puriscal & 6 & 6 & 48 & 0 & 0 & 0 & 60 \\
\hline San José & 1026 & 18 & 144 & 6 & 0 & 10 & 1204 \\
\hline
\end{tabular}


Cuadro 4. Distribución de animales activos Bos indicus con certificado de registro genealógico en Costa Rica para el año 2018, según raza y región socio-económica. San José, Costa Rica. 2018.

Table 4. Distribution of Bos indicus active animals with a certificate of genealogical registration in Costa Rica for the year 2018 , according to breed and socio-economic region. San José, Costa Rica. 2018.

\begin{tabular}{|c|c|c|c|c|c|c|}
\hline Raza & Central & Chorotega & Pacífico Central & Brunca & Huetar Atlántica & Huetar Norte \\
\hline Brahman gris & 268 & 3883 & 476 & 1229 & 1581 & 755 \\
\hline Nelore & 137 & 1125 & 0 & 22 & 201 & 34 \\
\hline Brahman rojo & 49 & 249 & 14 & 96 & 56 & 239 \\
\hline Gyr & 8 & 87 & 54 & 0 & 204 & 98 \\
\hline Guzerat & 0 & 98 & 0 & 0 & 1 & 0 \\
\hline Indobrasil & 10 & 7 & 0 & 0 & 0 & 14 \\
\hline Total & 472 & 5449 & 544 & 1347 & 2043 & 1140 \\
\hline
\end{tabular}

Cuadro 5. Distribución porcentual de la progenie activa de toros de la raza Brahman gris de acuerdo al registro de origen del padre. San José, Costa Rica. 2018.

Table 5. Percentage distribution of the active progeny of Brahman Gray bulls breed, according to the father's record origin. San Jose, Costa Rica. 2018.

\begin{tabular}{lcccc}
\hline & n & $\begin{array}{c}\text { Distribución porcentual } \\
\text { Origen del padre }\end{array}$ & Progenie & $\begin{array}{c}\text { Distribución } \\
\text { porcentual }\end{array}$ \\
\hline Progenie & & & & \\
Registro nacional & 492 & 66,7 & 4977 & 60,8 \\
Registro extranjero & 246 & 33,3 & 3214 & 39,2 \\
Total & $\mathbf{7 3 8}$ & $\mathbf{1 0 0 , 0}$ & $\mathbf{8 1 9 1}$ & $\mathbf{1 0 0 , 0}$ \\
\hline
\end{tabular}

\section{Genealogía de los cinco toros más utilizados en las fincas de ganado Brahman gris de Costa Rica}

La frecuencia de uso de reproductores de la población activa de la raza Brahman gris, mostró que los cinco reproductores más utilizados fueron los toros JDH Wellington Manso 527/1 registro número 864762 ABBA, RS Volcán del Guachipelín 573/3 T.E. registro 01-37555, RS Héroe del Guachipelín 23/3 T.E. registro 01-30486, CR Ticaban 273/0 F.I.V registro número 01-42553 y JDH Sir Alamo Manso 126/7 registro número 839795 ABBA. La progenie de estos cinco reproductores correspondió al 8,94\% de la población total activa con 732 animales inscritos, donde 466 fueron hembras y 266 fueron machos.

El toro JDH Wellington Manso 527/1, fue el de mayor descendencia a nivel nacional, pero es de origen estadounidense, inscrito en la Asociación Americana de Criadores de Brahman (ABBA). Este reproductor contaba con un total de 194 hijos registrados y activos (correspondientes al 2,3\% de la población total), los cuales se encontraron distribuidos en veintitres ganaderías ubicadas en las cinco regiones del país (Figura 2).

Toro RS Volcán del Guachipelín 573/3 T.E., fue el segundo reproductor a nivel nacional de acuerdo con el número de descendientes, es un toro de origen nacional. Cuenta con 154 hijos registrados y activos (correspondiente al 1,9\% de la población total), y se encuentran distribuidos en diez ganaderías ubicadas en cuatro regiones del país. Este toro es línea de sangre Marri por parte de su padre; su tatarabuelo es el toro JDH Domino Manso 42/4 registro 683995, hasta llegar en su árbol genealógico al toro Manso 162. Su genealogía materna corresponde a una vaca nacional de línea de sangre Manso (por el toro Aristocrat Manso), su tatarabuelo es el toro JDH Mr Union Manso 455/3 (Figura 3). 
Cuadro 6. Frecuencia de uso y sexo de las crías de reproductores de la raza Brahman gris utilizados en Costa Rica. San José, Costa Rica. 2018.

Table 6. Frequency of use and sex of the breeding offspring of the Gray Brahman breed used in Costa Rica. San Jose, Costa Rica. 2018.

\begin{tabular}{|c|c|c|c|}
\hline \multirow{2}{*}{ Reg. Padre } & \multicolumn{2}{|c|}{ Total general } & \multirow{2}{*}{ \% Total población } \\
\hline & $\mathbf{H}$ & M & \\
\hline 864762 & 105 & 89 & 2,37 \\
\hline 01-37555 & 89 & 65 & 1,88 \\
\hline 01-30486 & 108 & 33 & 1,72 \\
\hline $01-42553$ & 86 & 42 & 1,56 \\
\hline 839795 & 78 & 37 & 1,40 \\
\hline 839863 & 70 & 41 & 1,35 \\
\hline 877888 & 54 & 56 & 1,34 \\
\hline 837246 & 53 & 55 & 1,32 \\
\hline 803613 & 60 & 41 & 1,23 \\
\hline 846561 & 73 & 25 & 1,20 \\
\hline 863835 & 48 & 31 & 0,96 \\
\hline 830355 & 63 & 15 & 0,95 \\
\hline 01-40901 & 50 & 27 & 0,94 \\
\hline 854004 & 48 & 28 & 0,93 \\
\hline 780619 & 60 & 15 & 0,92 \\
\hline 873262 & 49 & 25 & 0,90 \\
\hline $01-42022$ & 38 & 33 & 0,87 \\
\hline $01-40280$ & 44 & 22 & 0,81 \\
\hline $01-24104$ & 44 & 21 & 0,79 \\
\hline $01-24324$ & 57 & 8 & 0,79 \\
\hline 813956 & 48 & 14 & 0,76 \\
\hline 718788 & 37 & 24 & 0,74 \\
\hline 727046 & 40 & 19 & 0,72 \\
\hline $01-28645$ & 30 & 28 & 0,71 \\
\hline $01-46197$ & 25 & 33 & 0,71 \\
\hline 01-39952 & 30 & 27 & 0,70 \\
\hline 747549 & 36 & 21 & 0,70 \\
\hline $01-42335$ & 36 & 17 & 0,65 \\
\hline 907467 & 22 & 31 & 0,65 \\
\hline $01-40637$ & 26 & 25 & 0,62 \\
\hline $01-46692$ & 24 & 27 & 0,62 \\
\hline 809260 & 30 & 20 & 0,61 \\
\hline 855430 & 32 & 18 & 0,61 \\
\hline $01-33678$ & 29 & 19 & 0,59 \\
\hline $01-45659$ & 20 & 28 & 0,59 \\
\hline 01-35665 & 27 & 19 & 0,56 \\
\hline $01-22169$ & 37 & 7 & 0,54 \\
\hline $01-42417$ & 32 & 12 & 0,54 \\
\hline
\end{tabular}




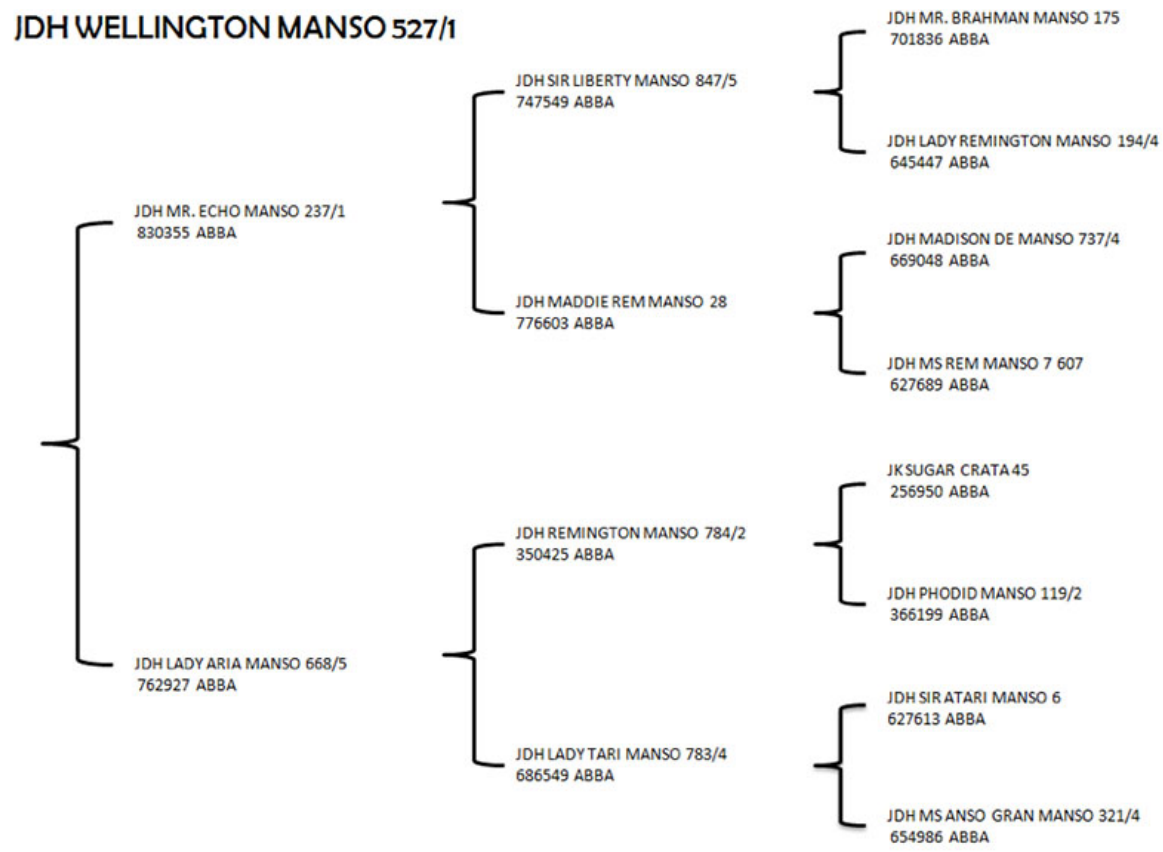

Figura 2. Árbol genealógico del reproductor raza Brahman gris, JDH Wellington Manso 527/1, registro número 864762 ABBA. San José, Costa Rica. 2018.

Figure 2. Family tree of the grey Brahman breed sire, JDH Wellington Manso 527/1 registration number 864762 ABBA. San José, Costa Rica, 2018

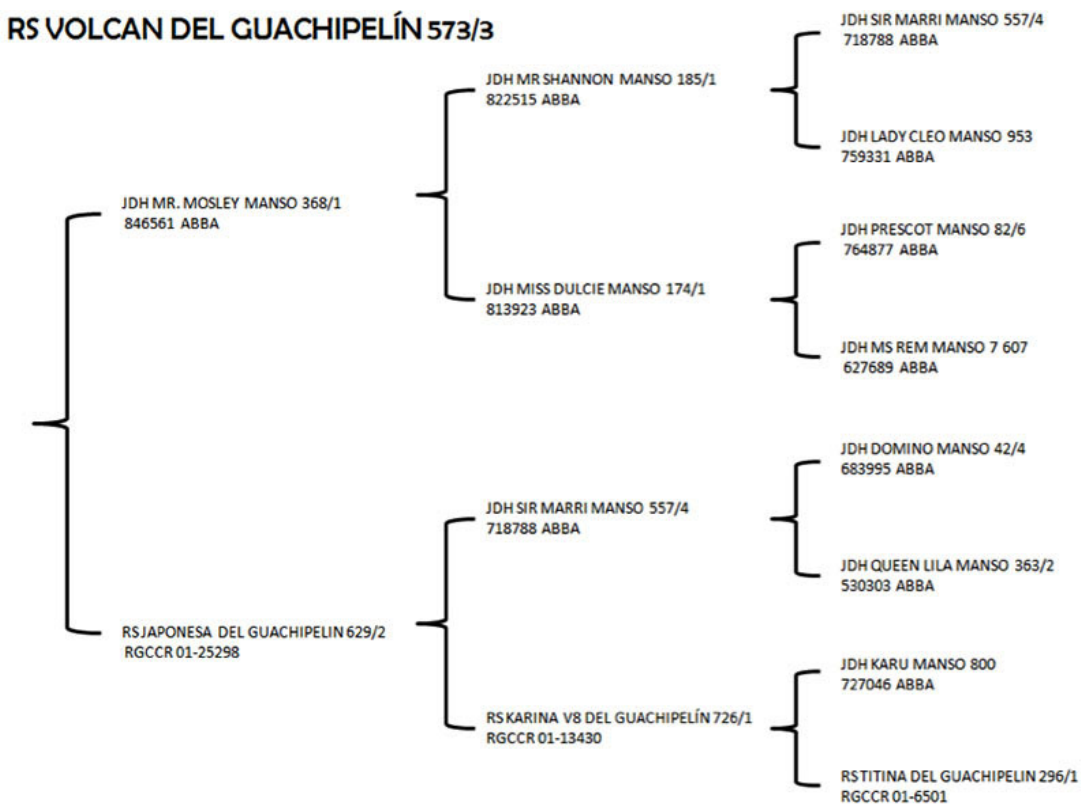

Figura 3. Árbol genealógico del reproductor raza Brahman gris, RS Volcán del Guachipelín 573/3 T.E. registro 01-37555. San José, Costa Rica. 2018.

Figure 3. Family tree of the gray Brahman breed sire, RS Volcan del Guachipelin 573/3 T.E. registration number 01-37555. San Jose, Costa Rica. 2018. 
El toro RS Heroé del Guachipelín 23/3, fue el tercer reproductor en número de descendencia inscrita y activa con 141 hijos (1,7 \% de la población total), es un toro de origen nacional y cuenta con hijos en veinte ganaderías, en cinco regiones del país. Su padre es proveniente de la línea de sangre Manso (por el toro Aristocrat Manso); su tatarabuelo es el toro JDH Odyssey Manso 479/2 registro 557739 ABBA. Su línea materna corresponde a una vaca nacional la cual, pese a que es hija de un toro nacional también es línea de sangre Manso (por el toro Aristocrat Manso), siendo su tatarabuelo el toro MR V8 202/3 registro 558653 ABBA (Figura 4).

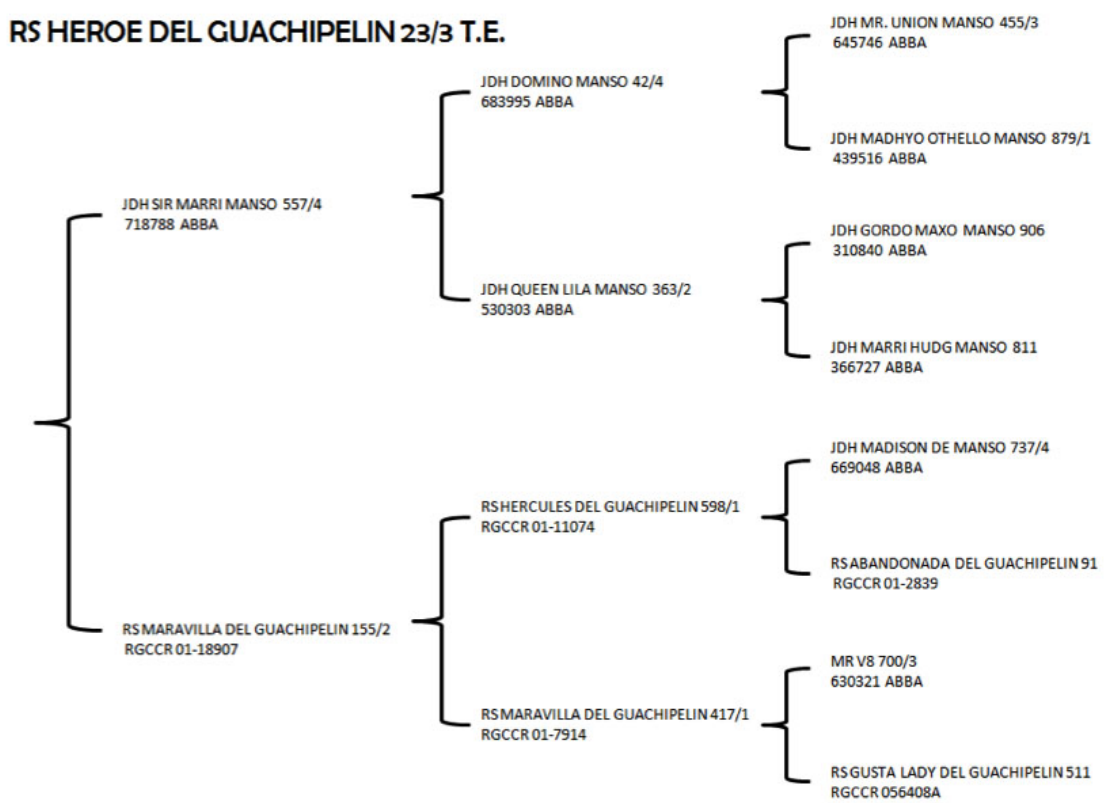

Figura 4. Árbol genealógico del reproductor raza Brahman gris, RS Héroe del Guachipelín 23/3 T.E. registro 01-30486. San José, Costa Rica, 2018.

Figure 4. Family tree of the grey Brahman breed sire, RS Heroe del Guachipelin 23/3 T.E. registration number 01-30486. San Jose, Costa Rica, 2018.

El toro CR Ticaban 273/0 F.I.V., fue el cuarto reproductor en número de descendencia inscrita y activa es de origen nacional. Cuenta con 128 hijos (correspondientes al 1,56 \% de la población total), ubicados en dos fincas en una sola región del país. Su padre es línea de sangre Madison que proviene a su vez de la línea de sangre Manso. Su tatarabuelo es el toro JDH Atari Manso 601/1 registro 359587 ABBA. Su línea materna es de origen guatemalteco, sin embargo, su madre es de línea de sangre Manso (toro Aristocrat Manso); su tatarabuelo es el toro JDH MrRha Ely Manso 615/6 registro número 340806 ABBA (Figura 5).

El toro JDH Sir Alamo Manso 126/7, fue el quinto reproductor en número de descendencia inscrita y activa con 115 hijos (correspondiente a 1,4\% de la población total), es de origen estadounidense, registro 839795 ABBA. Las crías de este toro se distribuyeron en diecisiete ganaderías, ubicadas en cinco regiones del país. Es línea de sangre Madison por parte del padre, su tatarabuelo es el toro JDH Atari Manso 601/1 registro 359587 ABBA. Su línea materna es Manso, su tatarabuelo es el toro JDH Odyssey Manso 479/2 registó 557739 ABBA (Figura 6). 


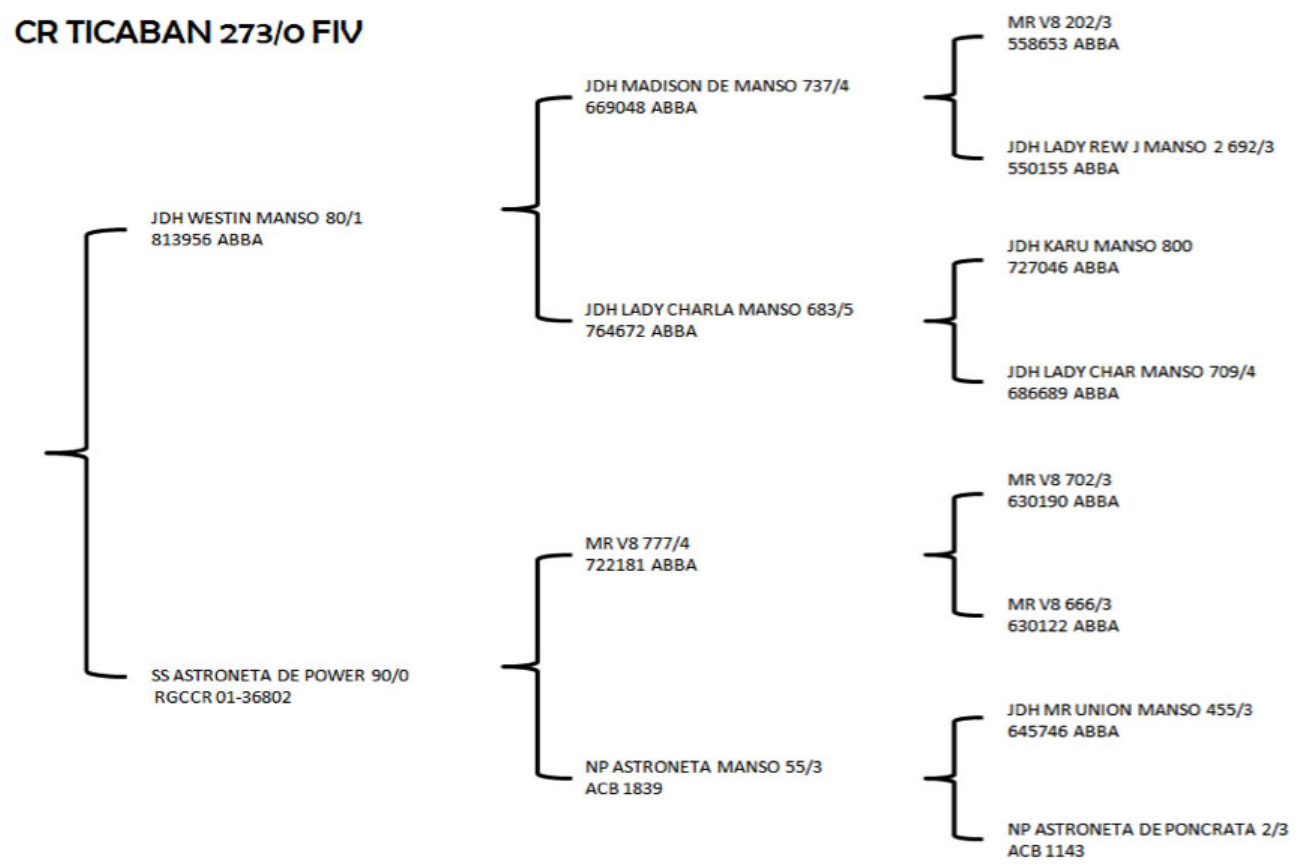

Figura 5. Árbol genealógico del reproductor raza Brahman gris, CR Ticaban 273/0 F.I.V registro número 01-42553. San José, Costa Rica, 2018.

Figure 5. Family tree of the grey Brahman breed sire, CR Ticaban 273/0 F.I.V registration number 01-42553. San Jose, Costa Rica, 2018.

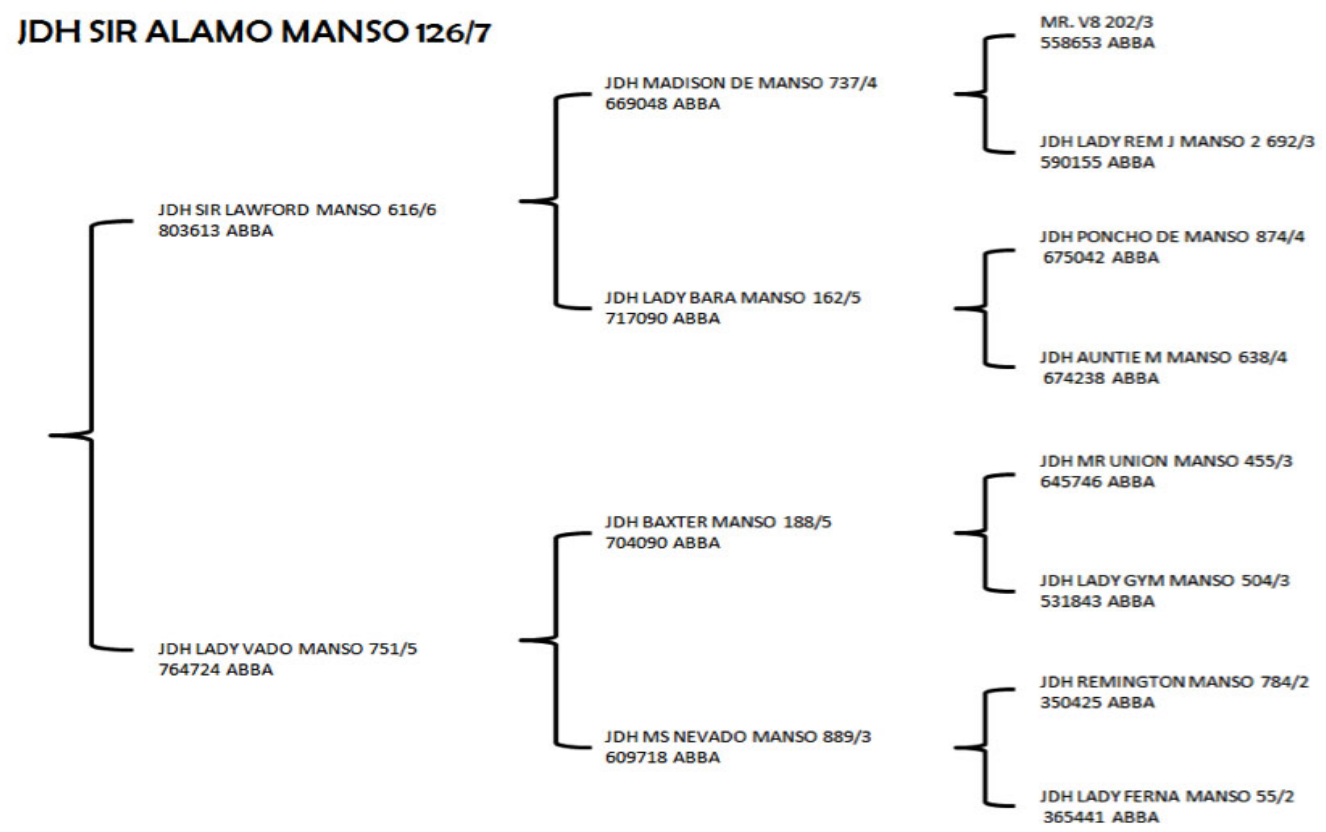

Figura 6. Árbol genealógico del reproductor raza Brahman gris, JDH Sir Alamo Manso 126/7 registro número 839795 ABBA. San José, Costa Rica. 2018.

Figure 6. Family tree of the grey Brahman breed sire, JDH Sir Alamo Manso 126/7 registration number 839795 ABBA. San Jose, Costa Rica. 2018. 


\section{Discusión}

Los datos encontrados demostraron la predominancia de la raza Brahman Gris con respecto al total de la población Bos indicus con certificado de registro genealógico. En este estudio, se utilizaron diferentes poblaciones y metodologías, que los utilizados por Madrigal y Fallas (2013), para la encuesta ganadera realizada en el año 2012, sin embargo, los resultados fueron similares, por ejemplo, Madrigal y Fallas (2013) encontraron que la raza Brahman estaba presente en el 77,7 \% del total de razas que conforman los sistemas de producción de carne y en el 76,9 \% en los sistemas de selección y pie de cría en Costa Rica, en este estudio la raza Brahman correspondió al $76,8 \%$ de toda la población cebuína registrada.

Independientemente de la raza, las hembras representaron el mayor porcentaje de la población con el 71,0\% (Cuadro 1), que mostró una relación macho - hembra de 1:3,3. Según el Censo Nacional Agropecuario 2014 (INEC, 2015), a nivel nacional se contaba con 882887 hembras bovinas (69 \% de la población), lo cual, coincide con el porcentaje encontrado en el presente estudio. Este mayor número de hembras activas puras con respecto a los machos, se puede atribuir a varios factores, entre ellos, algunas ganaderías realizaban una selección previa en los machos que son reportados e inscritos en ASOCEBÚ, sin embargo, el factor principal fue que un número de machos con certificado de registro genealógico fueron vendidos en edad de pubertad como reproductores a ganaderías de cría comercial, siendo este uno de los fines principales de la crianza de animales puros con certificado de registro, por tanto, no formaron parte de la población activa sobre la cual se realizó el inventario.

El desbalance entre la población activa de hembras y machos adultos, las frecuencias de nacimiento de machos y hembras inscritas en ASOCEBÚ fueron proporcionales, cercanas al $50 \%$ de cada uno, por ejemplo, en el periodo de enero a junio del 2018 la distribución porcentual de registros para la raza Brahman gris fue de 49,6\% en hembras y $50,4 \%$ en machos, para el total de razas inscritas fue de $54 \%$ y $46 \%$ en hembras y machos, respectivamente.

Respecto al tamaño de las fincas, en el caso de la encuesta ganadera realizada por CORFOGA en el año 2012, el mayor porcentaje de población del hato nacional $(28,4 \%)$ se encontraba distribuido en fincas de 30 a 84 cabezas de animales; sin embargo, un 16,7 \% de la población estaba concentrada en 556 fincas mayores a 300 cabezas de animales (Madrigal y Fallas, 2013). Mientras que según el Censo Nacional Agropecuario un 27,1\% del hato nacional estuvo en fincas mayores a 200 cabezas de animales (INEC, 2015). En el presente estudio, más del $50 \%$ de los animales registrados pertenecían a diez criadores, esto muestra una distribución diferente de población animal para criadores de ganado comercial y ganado cebú con certificado de registro genealógico.

El hato de ganado puro cebuino se encontraba distribuido en las siete provincias del país, con predominio en la provincia de Guanacaste y consecuentemente en la región Chorotega. Los datos encontrados sobre la distribución por región socio-económica, difirieron con los datos reportados por Madrigal y Fallas (2013), donde se encontró que la mayor cantidad de ganaderías dedicadas a selección y pie de cría de ganado de carne se localizó en la región Brunca $(25,6 \%)$, seguida por la región Chorotega $(6,8 \%)$ y en tercer lugar la región Huetar Norte $(5,8 \%)$. La diferencia en los resultados podría atribuirse a que los datos analizados por Madrigal y Fallas (2013) fueron producto de un muestreo realizado con base en 51158 fincas ganaderas inscritas en el SIREA establecidas en el territorio nacional, donde no se realizó distinción entre ganadería comercial y cría de ganado puro con certificado genealógico.

No se obtuvieron datos publicados acerca del uso de machos reproductores en esta u otra raza en el país, estos son los primeros resultados al respecto, los cuales brindan una alerta sobre la necesidad de llevar un registro y control del uso de los padrotes para evitar cruces consanguíneos y mejorar la productividad de la ganadería. El Programa Nacional de Evaluación y Mejoramiento Genético realizó evaluación de animales Brahman y estimó diferencias esperadas de progenie (DEP`S) en diferentes rasgos de interés (CORFOGA, 2020), pero no se refirió al uso y manejo genético de los animales en las fincas.

El análisis de la genealogía de los cinco reproductores más utilizados de la raza Brahman gris, evidenció que los mismos cuentan con ancestros en común, se presume que exista consanguinidad en la raza Brahman costarricense. 
Esta sospecha es congruente con los resultados reportados por Campos (2017), quien evalúo la diversidad genética en bovinos Brahman registrados en ASOCEBÚ Costa Rica, encontró un porcentaje de consanguinidad promedio de $4 \%$, el déficit de heterocigotos y la tendencia hacia la homocigosidad que encontró también lo confirmaron. Por su parte, Martínez et al. (2015), realizaron el primer estudio de variabilidad genética entre subpoblaciones raciales bovinas en Costa Rica, incluyeron animales puros y comerciales de la raza Brahman, al igual que Campos (2017), encontraron un déficit de heterocigotos. Ambos estudios atribuyen los resultados a prácticas de manejo como el uso excesivo de sementales, selección de reemplazos de un mismo padre y control en los apareamientos, con base en la selección artificial realizada por los criadores; también encontraron un fuerte impacto de los genes de ganado puro Brahman sobre los genes de ganado Brahman comercial (Martínez et al., 2015).

Al revisar la genealogía de los toros, se observó parentesco entre los animales de diferentes criadores. Un ejemplo fue el toro con mayor número de crías en el país, JDH Wellington Manson 527/1, su línea de sangre es Dakota por parte del padre; el abuelo paterno es el toro JDH Liberty Manso 847/5 registro 747549 ABBA. Respecto a su línea materna, es línea de sangre Manso (descendencia del toro Aristocrat Manso), su abuelo materno es el toro JDH Remington 784/2 el cual es un toro fuertemente consanguíneo sobre el toro Rex A Manso (Rueda, 2015), al contar en su línea paterna con el toro +Sugarland's Rexcrata 1229/3 como bisabuelo, siendo el padre tanto de la abuela como del abuelo. El tatarabuelo materno del JDH Wellington Manso 527/1 es el toro JDH IW S Rexcrata 402 registro 221717 ABBA, hasta llegar, en su árbol genealógico, al toro Manso 162, considerado el toro más importante en la formación de la raza Brahman. Según un artículo publicado por la revista Brahman (The Brahman Journal) (ABBA, 2017), este reproductor se encuentra entre los toros que tuvieron mayor descendencia registrada en la ABBA entre el 1 de enero de 2012 y el 31 de diciembre de 2016.

Los ganaderos comerciales pueden comprar en diferentes fincas de ganado puro, animales que están emparentados, por lo que es probable que a nivel de finca comercial, el grado de consanguinidad aumente. Es necesario que en Costa Rica se lleve un control adecuado de los apareamientos para evitar la consanguinidad y que no suceda, como en otros países, que la raza Brahman sea sometida a factores como selección artificial dirigida, reproducción endogámica, efectos de deriva genética y migración de ejemplares entre fincas y países (Novoa yUsaquén, 2006). Esto ha hecho que este proceso de selección haya aumentado el grado de consanguinidad (Gómez et al., 2013). La información precisa del pedigrí es esencial para el desarrollo de los programas de selección y raza, así como para mejorar la productividad en la industria animal (Cervini et al., 2006).

\section{Conclusiones}

La población de ganado cebuino con certificado de registro genealógico en Costa Rica se distribuye a lo largo de todo el país. La región Chorotega tuvo el mayor número de animales.

A nivel nacional se registraron animales provenientes de seis razas cebuinas, siendo Brahman gris la predominante, donde el mayor porcentaje de reproductores es de origen nacional. Aunque los ganaderos comerciales compren toros de diferentes ganaderías de ganado puro, pueden estar incorporando los mismos genes por los ancestros comunes de los progenitores originales utilizados por los criadores de ganado puro.

El análisis de la frecuencia de uso de reproductores en la raza Brahman mostró la necesidad de revisar los cruzamientos que se realizan en las fincas, a fin de minimizar el cruce consanguíneo. Así como realizar evaluaciones similares en otras razas utilizadas en el país.

Se sugiere que los censos o encuestas pecuarias realizadas por organizaciones gubernamentales incluyan de manera específica la información no solo del ganado comercial, sino también de la población de ganado puro con registro genealógico de las diferentes razas en el país. 


\section{Agradecimientos}

Los autores agradecen a la Asociación de Criadores de Ganado Cebú de Costa Rica por brindar las facilidades para el desarrollo de este proyecto.

\section{Literatura citada}

ABBA (American Brahman Breeders Association). 2017. Most influential gray herd sires in the last five years. The Brahman Journal, TX, USA. https://brahmanjournal.com/cattle/most-influential-gray-herd-sires-in-the-last-five-years (accessed Sep.25, 2017).

ASOCEBÚ (Asociación de Criadores de Ganado Cebú de Costa Rica). 2017. Raza Brahman. ASOCEBÚ, CRI. http://asocebucr. com/razas/\#raza-brahman (consultado 16 set. 2017).

ASOCEBÚ (Asociación de Criadores de Ganado Cebú de Costa Rica). 2020. Creación del registro genealógico. ASOCEBÚ, CRI. http://asocebucr.com/cebu-en-cr/\#creaci\%C3\%B3n-del-registro-geneal\%C3\%B3gico (consultado 25 mar. 2020).

Campos, J.A. 2017. Uso de marcadores genéticos para análisis de diversidad genética y pruebas de verificación de identidad y paternidad en bovinos Brahman registrados en Costa Rica. Tesis MSc., Universidad Nacional, CRI.

Campos, J., B. Vargas, J. Camacho, y A. Cruz. 2018. Pruebas de identidad y paternidad en ganado Brahman registrado de Costa Rica. Agron. Costarricense 42(1):49-62. doi:10.15517/rac.v42i1.32197

Cervini, M., F. Henrique-Silva, N. Mortari, y E. Matheucci. 2006. Genetic variability of 10 microsatellite markers in the characterization of Brazilian Nellore cattle (Bosindicus). Genet. Mol. Biol. 29:486-490. doi:10.1590/S141547572006000300015

CORFOGA (Corporación de Fomento Ganadero). 2000. Análisis del censo ganadero 2000. Ministerio de Agricultura y Ganadería, CRI. http://www.mag.go.cr/biblioteca_virtual_animal/censo-ganadero-2000.pdf (consultado 16 set. 2017).

CORFOGA (Corporación de Fomento Ganadero). 2020. Programa nacional de evaluación y mejoramiento genético. San José, CRI. https://www.corfoga.org/mejoramiento-genetico/ (consultado 17 mar. 2020).

Gómez, Y.M., M. Fernández, D. Rivera, G. Gómez, and J.E. Bernal. 2013. Genetic characterization of Colombian Brahman cattle using microsatellites markers. Russ. J. Genet. 49:737-745. doi:10.1134/S1022795413070041

INEC (Instituto Nacional de Estadística y Censo). 2015. VI Censo nacional agropecuario. INEC, San José, CRI.

Loftus, R.T., D.E. MacHugh, D.G. Bradley, P.M. Sharp, and P. Cunningham. 1994. Evidence for two independent domestications of cattle. Proc. Natl. Acad. Sci. 91:2757-2761. doi:10.1073/pnas.91.7.2757

Madrigal, J., y M. Fallas. 2013. Informe encuesta ganadera 2012. CORFOGA, San José, CRI.

Martínez, M., B. Vargas, J.M. Cordero, I. Chacón, y B. León. 2015. Diversidad Genética entre subpoblaciones raciales bovinas de Costa Rica. Agron. Costarricense 39(2):33-45.

Novoa, M.A., y W. Usaquén. 2006. Diversidad genética de la población colombiana de ganado Cebú Brahman Americano Bos indicus (Bovidae). Acta Biol. Colomb. 11:112.

Quirós, E. 2006. Historia de la Ganadería Bovina en Costa Rica. CORFOGA, San José, CRI.

Presidente de la República, y MAG (Ministerio de Agricultura y Ganadería). 1989. Decreto Rjrcutivo No19400-MAG: Traspasa registro genealógico de ganado a Asociación de Productores y Criadores de Ganado Procuraduría General de la República, 
CRI. http://www.pgrweb.go.cr/scij/Busqueda/Normativa/Normas/nrm_texto_completo.aspx?param1=NRTC\&nValor1=1 \&nValor2 $=4133 \&$ nValor3 $=4378 \&$ strTipM=TC (consultado 15 mar. 2020).

Rueda, J. 2015. Historia y líneas de sangre de la raza Brahman. Memoria del Seminario técnicas de selección y juzgamiento (San José, Costa Rica). ASOCEBÚ. San José, CRI.

Takeshima, S., C. Corbi-Botto, G. Giovambattista, and Y. Aida. 2018. Genetic diversity of BoLA-DRB3 in South American Zebu cattle populations. BMC Genetics 19:33. doi:10.1186/s12863-018-0618-7

Vélez, G. 2016. ¿Por qué utilizar ganado Cebú en el trópico? Tipo impreso Ltda., Bogotá, COL. 